\title{
Controlled Oxygen Doping Of Gan Using Plasma Assisted Molecular-Beam Epitaxy
}

\author{
A.J.Ptak \\ L. J. Holbert \\ L. Ting \\ C. H. Swartz \\ M. Moldovan \\ See next page for additional authors
}

Follow this and additional works at: https://researchrepository.wvu.edu/faculty_publications

\section{Digital Commons Citation}

Ptak, A. J.; Holbert, L. J.; Ting, L.; Swartz, C. H.; Moldovan, M.; Giles, N. C.; and Myers, T. H., "Controlled Oxygen Doping Of Gan Using Plasma Assisted Molecular-Beam Epitaxy" (2001). Faculty Scholarship. 926.

https://researchrepository.wvu.edu/faculty_publications/926 
Authors

A. J. Ptak, L. J. Holbert, L. Ting, C. H. Swartz, M. Moldovan, N. C. Giles, and T. H. Myers 


\title{
Controlled oxygen doping of GaN using plasma assisted molecular-beam epitaxy
}

\author{
A. J. Ptak, L. J. Holbert, L. Ting, C. H. Swartz, M. Moldovan, N. C. Giles, \\ and T. H. Myers ${ }^{\text {a) }}$ \\ Department of Physics, West Virginia University, Morgantown, West Virginia 26506
}

P. Van Lierde, C. Tian, R. A. Hockett, and S. Mitha

Evans Analytical Group, Charles Evans and Associates, Sunnyvale, California 94086

A. E. Wickenden, ${ }^{\text {b) }}$ D. D. Koleske, and R. L. Henry

Laboratory for Advanced Material Synthesis, Code 6861, Electronics Science and Technology Division, Naval Research Laboratory, Washington, DC 20375

(Received 7 May 2001; accepted for publication 16 July 2001)

\begin{abstract}
High-quality (0001) and (000) $\overline{1}$-GaN films were grown by plasma-assisted molecular-beam epitaxy to study the dependence of oxygen incorporation on polarity and oxygen partial pressure. Oxygen incorporates at a rate ten times faster on nitrogen-polar GaN than on the Ga polarity. Oxygen doping is controllable, reproducible, and produces low compensation material up to concentrations of at least $10^{18} \mathrm{~cm}^{-3}$ with higher levels showing significant compensation. Layers containing oxygen at levels above $10^{22} \mathrm{~cm}^{-3}$ exhibit severe cracking while oxygen concentrations less than $10^{21} \mathrm{~cm}^{-3} \mathrm{do}$ not introduce significant strain. The oxygen incorporation rate has a weak dependence on Ga overpressure during Ga-stable growth but dramatically increases for conditions approaching N-stable growth. ㅇ 2001 American Institute of Physics. [DOI: 10.1063/1.1403276]
\end{abstract}

Many groups have studied the role of oxygen in GaN, but most studies have focused on unintentionally doped material with oxygen supplied either by an "uncontrolled" system background or from outdiffusion from the sapphire substrate. Also of importance, and something that has not received the proper attention, is the effect of surface polarity on the incorporation of oxygen. Zywietz et al. ${ }^{1}$ used firstprinciples calculations to predict that, for typical Ga-stable growth conditions, the Ga-polar surface would adsorb lower amounts of oxygen than the N-polar surface. A secondary ion mass spectrometry (SIMS) study of Ga- and N-polar GaN grown by metalorganic chemical vapor deposition (MOCVD) by Sumiya et al. ${ }^{2}$ found about 170 times more oxygen for $\mathrm{N}$-polar growth. However, N-polar material grown by MOCVD is typically of poor quality, raising the uncertainty that poor structure could be the cause of the increased incorporation. It is also not clear this result is directly applicable to the molecular-beam epitaxy (MBE) growth of GaN. In this letter, we present the results of a controlled study of oxygen incorporation in $\mathrm{GaN}$ using MBE.

The oxygen doped GaN layers were grown at West Virginia University (WVU) by rf plasma-assisted MBE using procedures described in prior studies. ${ }^{3}$ Ultrahigh purity oxygen gas $(99.998 \%)$ was introduced through a tube directed at the substrate using an ultrahigh vacuum leak valve and monitored using a residual gas analyzer. N-polarity GaN was obtained by nucleating GaN buffer layers directly on sapphire under heavily Ga-rich conditions. ${ }^{4}$ Incorporation in Gapolarity GaN was studied by growth on semi-insulating

${ }^{a)}$ Electronic mail: tmyers@wvu.edu

b) Also at: Army Research Laboratory, AMSRL-SE-RL, Adelphi, Maryland 20783.
MOCVD GaN templates on A-plane sapphire substrates grown at the Naval Research Laboratory. Transmission electron microscopy studies on similar layers grown under identical conditions at WVU indicated that our growth procedure produces N-polar and Ga-polar layers of comparable quality, with typical dislocation densities in the low $10^{9} \mathrm{~cm}^{-3} .^{5}$

Temperature-dependent Hall effect measurements were performed using indium to form ohmic contacts with the standard van der Pauw geometry. Measurements routinely included several current levels at each temperature to ensure contact resistance was not an issue, particularly for the lower doped samples. The use of semi-insulating MOCVD templates resolved the difficulties encountered in measuring low carrier concentration material when using conducting templates. Room temperature Raman spectroscopy was performed using a Jobin-Yvon Ramanor U-1000 micro-Raman system excited by $476 \mathrm{~nm}$ laser light. SIMS measurements at Evans Analytical Group, Charles Evans and Associates (Sunnyvale, CA) determined the concentration of oxygen in the films using a Cameca ion microprobe.

Figure 1 shows a SIMS measurement of oxygen incorporation for a N-polar step-doped sample. During growth, the doped regions were created through adjusting the leak valve to attain the proper oxygen partial pressure. Approximately $0.25 \mu \mathrm{m}$ of doped material was grown in each step followed by closing the oxygen valve to grow a $0.5 \mu \mathrm{m}$ thick undoped spacer. A clear dependence of the concentration of oxygen, $[\mathrm{O}]$, is seen with increasing oxygen partial pressure. The steps are very sharp (less than $8 \mathrm{~nm}$ decade) for N-polarity growth. Similar Ga-polar structures grown on MOCVD templates exhibited the same trend for incorporation with oxygen partial pressure, but the steps were not as abrupt (approximately $88 \mathrm{~nm} /$ decade).

Oxygen doping to produce $n$-type material was controllable and reproducible under Ga-stable growth conditions us- 


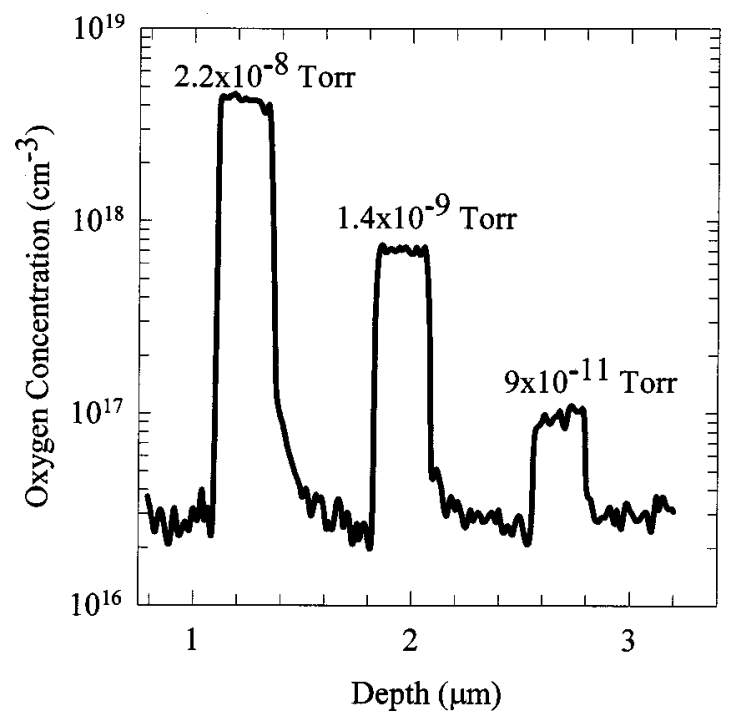

FIG. 1. SIMS measurement showing oxygen incorporation in a N-polar step-doped sample is presented. The partial pressure of oxygen used for each step is indicated.

ing $\mathrm{O}_{2}$ as the dopant source for concentrations up to $10^{18} \mathrm{~cm}^{-3}$. Figure 2 compares the least-squares fit to the oxygen concentrations as determined by SIMS from the stepdoped layers to room-temperature Hall effect measurements of carrier concentration from uniformly doped layers. The lines representing the SIMS data have been extrapolated below the SIMS detection limit. N-polar GaN incorporates about one out of every fifty available oxygen atoms into the growing layer based on measured partial pressures. Incorporation in Ga-polar GaN is less effective by about an order of magnitude. The two oxygen incorporation trend lines for Gapolar growth indicated in Fig. 2 were generated for different Ga overpressures.

The room temperature carrier concentrations determined by Hall effect measurements are consistent with oxygen acting as a shallow donor that appears uncompensated up to concentrations of $\sim 10^{18} \mathrm{~cm}^{-3}$. The oxygen incorporation predicted from the SIMS calibration and the measured carrier concentration were in good agreement for both polarities, with all measurements agreeing to within a factor of two. Interestingly, measured carrier concentrations from our unintentionally doped Ga-polar material correlates nicely with the extrapolated oxygen concentration predicted by the SIMS calibration based on the residual background partial pressure of oxygen in our system during growth (approximately $4-5 \times 10^{-12}$ Torr). This strongly suggests that our residual $n$-type carrier background is primarily due to oxygen. The SIMS and Hall effect results provide consistent evidence of the influence of polarity on the incorporation of oxygen.

The temperature-dependent Hall effect data for a Ga polar sample shown in Fig. 3 indicates a room temperature mobility of $\sim 500 \mathrm{~cm}^{2} / \mathrm{V} \mathrm{s}$ and a peak mobility at low temperature of $\sim 1500 \mathrm{~cm}^{2} / \mathrm{V}$ s. Importantly, carrier concentration data obtained for O-doped layers such as that shown in Fig. 3 is consistent with a single donor in relatively uncompensated material. While care must be taken in interpreting fits to the full charge-balance equation, this result is again consistent with the close correlation between Hall and SIMS results indicated in Fig. 1. Direct analysis of the data shown Downloaded 30 Nov 2001 to 157.182.108.112. Redistribution subje

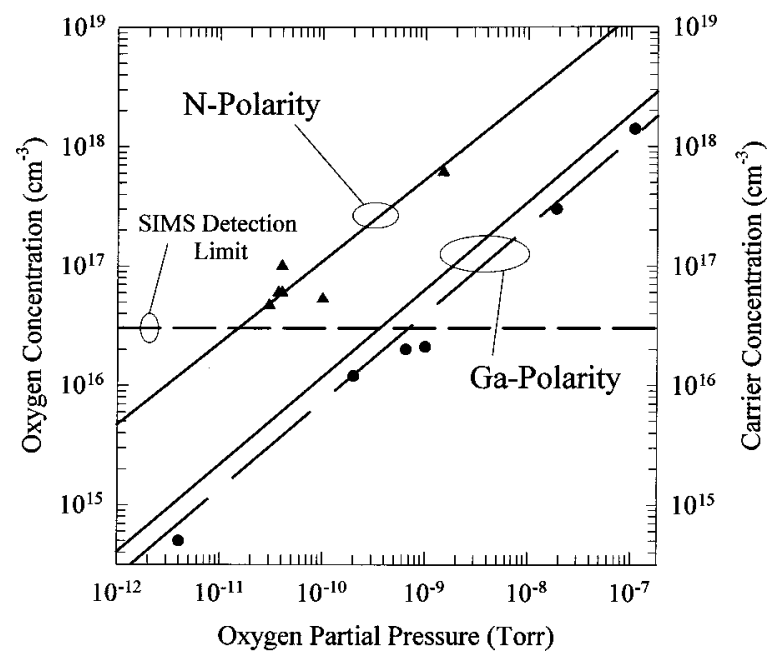

FIG. 2. SIMS measurement of oxygen concentration (lines) and room temperature Hall carrier concentrations (points) vs oxygen partial pressure is shown. The lines represent a least-squares fit to SIMS oxygen concentration for constant Ga-overpressure (solid: $1.6 \times 10^{-6}$ Torr BEP and dashed: 1.0 $\times 10^{-6}$ Torr BEP). N-polar Hall results were for layers grown under a $\mathrm{Ga}$ overpressure of $1.6 \times 10^{-6}$ Torr BEP while Ga-polar Hall results were for a Ga-overpressure of $1.0 \times 10^{-6}$ Torr BEP.

in Fig. 3 indicates a thermal activation energy of $30 \mathrm{meV}$ for this oxygen doped sample. When carrier screening is taken into account, ${ }^{6}$ a donor energy of $35 \mathrm{meV}$ is indicated. Analyses of all temperature-dependent Hall measurements to date on layers with carrier concentrations between $10^{16}$ and $10^{18} \mathrm{~cm}^{-3}$ are consistent with oxygen forming an uncompensated shallow donor with a donor energy level between 35 and $39 \mathrm{meV}$. Similar Hall activation energies were observed for oxygen doping on both polarities of GaN. The maximum room temperature mobilities measured were around 500 $\mathrm{cm}^{2} / \mathrm{V} \mathrm{s}$ and occurred at doping levels of about $10^{16} \mathrm{~cm}^{-3}$. Mobility values dropped to $90 \mathrm{~cm}^{2} / \mathrm{V}$ s for the lowest carrier concentration measured, $\sim 5 \times 10^{14} \mathrm{~cm}^{-3}$, consistent with predictions of dislocation scattering for an estimated dislocation density in the low $10^{9} \mathrm{~cm}^{-2}$. $^{7}$ The mobility also steadily decreased with increasing oxygen concentration above $10^{16} \mathrm{~cm}^{-3}$, consistent with increased carrier scattering, with resulting mobilities slightly lower than the corresponding results for equivalent Si-doping in $\mathrm{GaN}^{8}$

During Ga-stable growth, oxygen incorporation exhibited only a small dependence on Ga overpressure. Incorporation increased about a factor of two upon changing the $\mathrm{Ga}$ beam-equivalent pressure (BEP) from 1.0 to 1.6 $\times 10^{-6}$ Torr. In contrast, oxygen incorporation was found to increase by up to four orders of magnitude for growth approaching $\mathrm{N}$-stable conditions (approximately 5.0 $\times 10^{-7}$ Torr Ga BEP). The dramatic increase in [O] for a given partial pressure as growth approaches $\mathrm{N}$-stable conditions is indicated by the data contained in Table I. The sharp increase with decreasing Ga flux makes oxygen incorporation difficult to control under these conditions, unlike Gastable growth.

Significant compensation occurs for concentrations above $10^{21} \mathrm{~cm}^{-3}$ as seen by comparing the oxygen incorporation and the carrier density in Table I. The samples with the highest [O] also exhibited significant cracking. Similar cracking is observed for heavy $\mathrm{Si}$ doping due to tensile stress to AIP license or copyright, see http://ojps.aip.org/aplo/aplcr.jsp 


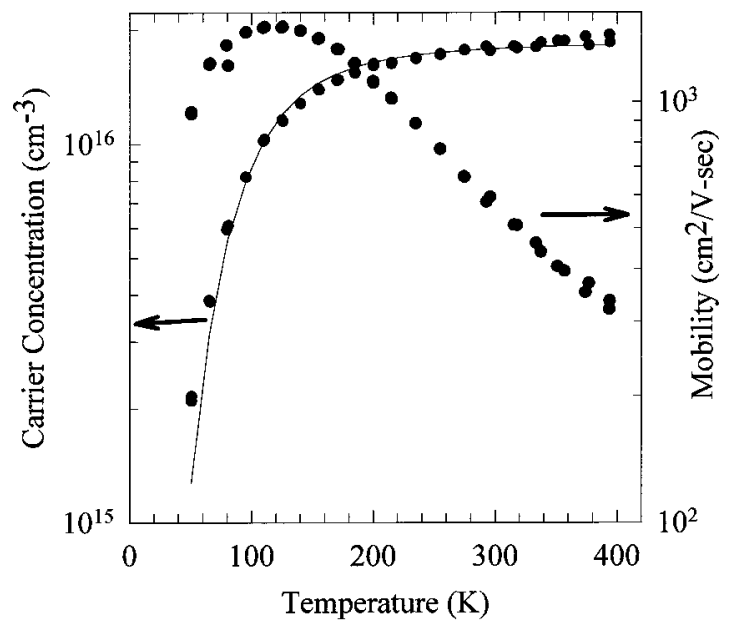

FIG. 3. Temperature-dependent Hall effect measurement of an oxygen doped Ga-polar sample is shown. The line is the fit to the full chargebalance equation assuming a single donor with a potential compensating acceptor with results indicating the sample is reasonably uncompensated.

occurring for Si levels above $2 \times 10^{19} \mathrm{~cm}^{-3} .{ }^{9}$ Raman spectroscopy can be used to measure stress in GaN (Ref. 9) by monitoring shifts in the $E_{2}$ Raman peak energy (at $\sim 569$ $\mathrm{cm}^{-1}$ ). For most Ga-polar layers, the $E_{2}$ peak was measured for the MOCVD template prior to growth for comparison with that measured for the composite system of $1 \mu \mathrm{m}$ doped MBE on about $2 \mu \mathrm{m}$ of MOCVD GaN. For N-polar layers, comparison was made between undoped and oxygen doped layers of the same thickness. Interestingly, no evidence of increased strain was measured for either polarity for [O] up to $\sim 3 \times 10^{21} \mathrm{~cm}^{-3}$. This result is consistent with calculations indicating the tensile strain observed in highly $n$-type Sidoped $\mathrm{GaN}$ is not due to electronic effects ${ }^{9}$ or else a similar large strain would also be observed in these highly doped layers. Some drastic effect does occur, however, for [O] above this level as all layers with [O] higher than $10^{22} \mathrm{~cm}^{-3}$ exhibit significant cracking.

Oxygen incorporation trends are consistent with the prediction that the formation energy of substitutional oxygen should decrease with an increasing III/V ratio for Ga-stable conditions. ${ }^{10}$ As discussed, the incorporation of oxygen shows a dramatic increase, by up to four orders of magnitude, upon approaching $\mathrm{N}$-stable growth conditions. Unlike most semiconductors, the growing $\mathrm{GaN}$ surface does not relax by forming dimers or other complex structures to reduce surface dangling bonds. Instead, these bonds are saturated by the formation of one or more Ga adlayers. ${ }^{11}$ Thus, the surface becomes highly reactive under growth conditions hindering formation of the Ga adlayer. The resulting surface dangling bonds strongly increase the adsorption of oxygen, explaining the increased oxygen affinity ${ }^{1}$ as $\mathrm{N}$-stable conditions are approached. Indeed, for lower $\mathrm{Ga}$ coverage and lower $\mathrm{O}_{2}$ flux, both polarities are predicted to be equally reactive to oxygen incorporation. ${ }^{1}$ It is the presence of the Ga adlayer that significantly reduces the adsorption and incorporation of oxygen, with a more pronounced effect on the Ga-polarity sur-
TABLE I. Growth conditions and results for oxygen doped layers are shown.

\begin{tabular}{ccccc}
\hline \hline Polarity & $\begin{array}{c}\text { Ga BEP } \\
(\text { Torr })\end{array}$ & $\begin{array}{c}P_{\mathrm{O}_{2}} \\
(\text { Torr })\end{array}$ & $\begin{array}{c}{[\mathrm{O}]^{\mathrm{a}}} \\
\left(\mathrm{cm}^{-3}\right)\end{array}$ & $\begin{array}{c}n^{\mathrm{b}} \\
\left(\mathrm{cm}^{-3}\right)\end{array}$ \\
\hline $\mathrm{N}$ & $1.6 \times 10^{-6}$ & $1 \times 10^{-10}$ & & $5.4 \times 10^{16}$ \\
$\mathrm{~N}$ & $8.3 \times 10^{-7}$ & $1 \times 10^{-10}$ & & $6.3 \times 10^{17}$ \\
$\mathrm{~N}$ & $1.8 \times 10^{-6}$ & $3.0 \times 10^{-7}$ & & $3.2 \times 10^{18}$ \\
$\mathrm{~N}$ & $9.2 \times 10^{-7}$ & $1.8 \times 10^{-7}$ & & $9.0 \times 10^{19}$ \\
$\mathrm{~N}$ & $5.0 \times 10^{-7}$ & $1.7 \times 10^{-7}$ & & $6.8 \times 10^{19}$ \\
$\mathrm{Ga}$ & $5.0 \times 10^{-7}$ & $6 \times 10^{-8}$ & $1.5 \times 10^{20}$ & \\
$\mathrm{Ga}$ & $1.6 \times 10^{-6}$ & $6 \times 10^{-8}$ & $1.6 \times 10^{18}$ & \\
$\mathrm{Ga}$ & $1.0 \times 10^{-6}$ & $6 \times 10^{-8}$ & $8.5 \times 10^{17}$ & \\
$\mathrm{Ga}$ & $9.5 \times 10^{-7}$ & $1.0 \times 10^{-7}$ & & $1.4 \times 10^{18}$ \\
$\mathrm{Ga}$ & $4.8 \times 10^{-7}$ & $5.0 \times 10^{-8}$ & $2.6 \times 10^{21}$ & $4.0 \times 10^{19}$ \\
$\mathrm{Ga}$ & $4.9 \times 10^{-7}$ & $2.0 \times 10^{-7}$ & $1.5 \times 10^{22}$ & $8.4 \times 10^{19}$ \\
$\mathrm{Ga}$ & $4.9 \times 10^{-7}$ & $1.5 \times 10^{-7}$ & $2.5 \times 10^{22}$ & $3.0 \times 10^{20}$ \\
\hline \hline
\end{tabular}

${ }^{\mathrm{a}}$ Measured by SIMS.

${ }^{\mathrm{b}}$ Measured by Hall effect at $300 \mathrm{~K}$.

face. Therefore, it is important to grow high-quality GaN under sufficiently Ga-stable conditions to minimize the incorporation of background impurities such as oxygen.

In summary, a controlled study of oxygen incorporation in high-quality $\mathrm{GaN}$ grown by plasma-assisted MBE shows distinct surface polarity dependence, with the $(000 \overline{1})$ polarity incorporating about an order of magnitude more oxygen than the (0001) polarity. Oxygen can be an effective and controllable donor impurity. Low compensation, unstrained material with carrier concentrations up to $10^{18} \mathrm{~cm}^{-3}$ has been obtained although significant strain and electrical compensation do occur at higher levels. Oxygen shows an incorporation dependence on Ga overpressure during growth with a drastic incorporation increase when approaching $\mathrm{N}$-stable conditions likely due to the elimination of the Ga adlayers that exist on the surface during Ga-stable growth.

The work at WVU was supported by ONR Grant No. N00014-96-1-1008 and ONR Grant No. N00014-99-1-0520, both monitored by Colin E. C. Wood.

${ }^{1}$ T. K. Zywietz, J. Neugebauer, and M. Scheffler, Appl. Phys. Lett. 74, 1695 (1999).

${ }^{2}$ M. Sumiya, K. Yoshimura, K. Ohtsuka, and S. Fuke, Appl. Phys. Lett. 76, 2098 (2000).

${ }^{3}$ A. J. Ptak, T. H. Myers, L. T. Romano, C. G. Van de Walle, and J. E. Northrup, Appl. Phys. Lett. 78, 285 (2001).

${ }^{4}$ T. H. Myers, L. S. Hirsch, L. T. Romano, and M. R. Richards-Babb, J. Vac. Sci. Technol. B 16, 2261 (1998).

${ }^{5}$ L. T. Romano, Xerox Palo Alto Research Center, Palo Alto, CA (Unpublished).

${ }^{6}$ D. Meister, M. Bohm, M. Topf, W. Kriegseis, W. Burkhardt, I. Dirnstorfer, S. Rosel, B. Farangis, B. K. Meyer, A. Hoffmann, H. Siegle, C. Thomsen, J. Christen, and F. Bertram, J. Appl. Phys. 88, 1811 (2000).

${ }^{7}$ H. M. Ng, D. Doppalapudi, T. D. Moustakas, N. G. Weimann, and L. F. Eastman, Appl. Phys. Lett. 73, 821 (1998).

${ }^{8}$ S. N. Mohammad, A. A. Salvador, and H. Morkoc, Proc. IEEE 83, 1306 (1995).

${ }^{9}$ L. T. Romano, C. G. Van de Walle, J. W. Ager III, W. Gotz, and R. S. Kern, J. Appl. Phys. 87, 7745 (2000).

${ }^{10}$ C. G. Van de Walle, C. Stampfl, and J. Neugebauer, J. Cryst. Growth 189, 505 (1998).

${ }^{11}$ A. R. Smith, R. M. Feenstra, D. G. Greve, J. Neugebauer, and J. E. Nothrup, Phys. Rev. Lett. 79, 3934 (1997). 